\title{
SUPER-RESOLUTION FLUORESCENCE IMAGING OF THE SEEDING AND POLYMERIZATOIN OF THE HUNTINGTIN EXON 1 PROTEIN
}

Ya Zhou (1), Laurence J. Young (2), Amberley S. Stephens (2), Christian Landles (1), Clemens Kaminski (2), Gabriele S. Kaminski Schierle (2), Gillian P. Bates (1)

(1) UCL Huntington's Disease Centre and Sobell Dept. Motor Neuroscience, UCL Institute of Neurology, University College London, London WC1N 3BG, UK.

(2) Department of Chemical Engineering and Biotechnology, University of Cambridge, Pembroke Street, Cambridge CB2 3RA UK.

Background Huntington's disease (HD) is caused by the expansion of the CAG repeat in exon 1 of the gene coding for the huntingtin (HTT) protein. The mutant version of the polyglutamine tract can result in misfolding and aggregation of $\mathrm{N}$-terminal fragments of HTT under physiological conditions. Therefore, it's crucial to understand the mechanisms underlying the seeding and polymerization of HTT exon 1. However, the formation of HTT aggregates has been difficult to investigate by conventional fluorescence microscopy due to their small size. Recent advances in super-resolution microscope have made this possible by permitting researchers to investigate protein aggregation in much greater detail.

Aims To use highly novel super-resolution microscope techniques to understand the mechanisms underlying the seeding and polymerization of HTT exon 1

Methods/Techniques Purify native HTT exon 1 protein from newly designed constructs and use two-colour TIRF (total internal reflection fluorescence microscope)-SIM (Structured illumination microscopy) microscope to directly observe the growth of HTT exon 1 at the single fibril level.

Results/Outcome We have designed and cloned new constructs ideal for making native HTT exon 1 protein by precise protease cleavage and fluorescent dye labelling for microscope imaging. The characterization and application of these constructs will be presented.

Conclusions Our methods will enable us to directly visualize the dynamics of the HTT exon 1 aggregation and study the aggregation kinetics which is important to HD therapeutics. 